\title{
Prevalência das alterações sistêmicas dos pacientes atendidos pela Clínica de Odontologia do Centro Universitário Ruy Barbosa
}

Prevalence of systemic changes of patients administered by the Dentistry Clinic of Ruy Barbosa University Center Prevalencia de cambios sistémicos de pacientes administrados por la Clínica de Odontología del Centro de la Universidad Ruy Barbosa

Kaíque Carvalho de SouzA

Anna Raquel Gomes de Araújo QUEIROZ Rosane Borges DIAS

Daniele Veiga da Silva SIQUEIRA

Thaís Feitosa de Oliveira LEITÃo Virgínia Dias Uzêda e SILVA

Curso de Odontologia, Centro Universitário Ruy Barbosa Wyden - UNIRUY, 41730-101 Salvador - BA, Brasil

\section{Resumo}

Complicações médicas, apesar de raras, podem ocorrer durante o atendimento odontológico, os pacientes portadores de alterações sistêmicas são os mais propensos a esta ocorrência, portanto requerem um atendimento especial pelo cirurgiãodentista. Objetivo: Avaliar o perfil das condições sistêmicas dos pacientes atendidos na clínica de Odontologia do Centro Universitário Ruy Barbosa. Metodologia: Foi realizado o levantamento dos pacientes atendidos na Clínica de Odontologia do Centro Universitário Ruy Barbosa - Wyden no período de 2016.2-2019.1. Os dados foram coletados a partir dos prontuários clínicos dos pacientes que se submeteram a atendimento no serviço da Faculdade e foram originalmente preenchidos pelos alunos de Graduação dessa instituição totalizando 719 prontuários. Os prontuários preenchidos incorretamente ou com dados incompletos foram contabilizados como incompletos. Os resultados foram analisados descritivamente, por porcentagem na presença de alterações sistêmicas dos pacientes encontrados no prontuário. Resultados: Do total dos prontuários analisados as condições sistêmicas mais prevalentes foi a hipertensão com $81(27,55 \%)$ casos encontrados, seguido por anemia com 69 $(23,55 \%)$ casos, alergia medicamentosa com $41(17,9 \%)$ casos, diabetes e coagulopatia com $29(9,9 \%)$ casos cada encontrados. Conclusão: As alterações mais prevalentes foram a hipertensão, anemia, alergia medicamentosa e diabetes, assim, predominando nas mulheres e na faixa etária entre 40 a 49 anos. Sendo de fundamental importância a avaliação criteriosa dos pacientes durante a consulta odontológica para identificação de possíveis problemas que possam alterar o plano de tratamento.

Descritores: Doença; Comorbidade; Epidemiologia; Assistência Odontológica.

\section{Abstract}

Medical complications may occur during dental care, patients with systemic alterations are the most prone to this occurrence, so they require a special care by the dentist. Objective: To evaluate the profile of the systemic conditions of the patients attended at the Dentistry Clinic of the University Center Ruy Barbosa. Methodology: A survey was carried out of the patients attended at the Deny Clinic of the University Center Ruy Barbosa - Wyden in the period of 2016.2-2019.1. The data were collected from the medical records of patients who underwent care in the Faculty and were originally filled by the undergraduate students of this institution, totaling 719 medical records. Incorrectly completed or incomplete records were counted as incomplete. The results were analyzed descriptively, by percentage in the presence of systemic alterations of the patients found in the medical record. Results: The most prevalent systemic conditions were hypertension with $81(27.55 \%)$ cases, followed by anemia with 69 (23.55\%) cases, drug allergy with $41(17.9 \%)$ cases, diabetes and coagulopathy with 29 (9.9\%) cases each found. Conclusion: The most prevalent changes were hypertension, anemia, drug allergy and diabetes, thus predominating in women and in the age group between 40 and 49 years. It is the fundamental importance the careful evaluation of the patients during the dental consultation to identify possible problems that may alter the treatment plan.

Descriptors: Disease; Comorbidity; Epidemiology; Dental Care.

\section{Resumen}

Las complicaciones médicas, aunque raras, pueden ocurrir durante la atención dental, los pacientes con cambios sistémicos son los más propensos a esta ocurrencia, por lo tanto, requieren atención especial por parte del cirujano dental. Objetivo: evaluar el perfil de afecciones sistémicas de pacientes tratados en la Clínica de Odontología del Centro Universitario Ruy Barbosa. Metodología: La encuesta a pacientes tratados en la Clínica de Odontología del Centro Universitario Ruy Barbosa Wyden se realizó durante 2016.2-2019.1. Los datos se obtuvieron de los registros clínicos de pacientes que fueron atendidos en el servicio de la Facultad y fueron completados originalmente por estudiantes universitarios de esta institución por un total de 719 registros. Los registros completados incorrectamente o incompletos se contaron como incompletos. Los resultados se analizaron descriptivamente por porcentaje en presencia de cambios sistémicos en los pacientes encontrados en los registros médicos. Resultados: Del total de registros médicos analizados, las afecciones sistémicas más prevalentes fueron hipertensión con $81(27.55 \%)$ casos, seguida de anemia con 69 (23.55\%) casos, alergia a medicamentos con $41(17.9 \%)$ casos. , diabetes y coagulopatía con $29(9,9 \%)$ casos cada uno encontrado. Conclusión: Los cambios más prevalentes fueron hipertensión, anemia, alergia a medicamentos y diabetes, predominando así en mujeres y en el grupo de edad entre 40 y 49 años. Es de fundamental importancia la evaluación cuidadosa de los pacientes durante la cita dental para identificar posibles problemas que puedan cambiar el plan de tratamiento.

Descriptores: Enfermedad; Comorbilidad; Epidemiología; Atención Odontológica.

INTRODUÇÃO

As doenças crônicas não transmissíveis (DCNT) são as principais causas de mortes no mundo e têm gerado elevado número de mortes prematuras, perda de qualidade de vida com alto grau de limitação nas atividades de trabalho e de lazer, além de impactos econômicos para as famílias, comunidades e a sociedade em geral, agravando as iniquidades e aumentando a pobreza. As DCNT caracterizam-se por ter uma etiologia múltipla, muitos fatores de risco, longos períodos de latência, curso prolongado, origem não infecciosa e também por associarem-se a deficiências e incapacidades funcionais. Sua ocorrência é muito influenciada 
pelas condições de vida, pelas desigualdades sociais, não sendo resultado apenas dos estilos de vida ${ }^{1,2}$.

A saúde bucal e geral não pode ser desvinculada e, por este motivo, a Medicina e a Odontologia devem interagir de forma que a promoção de saúde seja o principal foco de atenção profissional. Essa filosofia subentende uma reforma do pensamento, ou seja, considerar a saúde como um sistema complexo de relações em que a integração entre as disciplinas tenha a saúde como o eixo do questionamento e o indivíduo como objetivo de ação ${ }^{3}$.

O número de pacientes necessitando de tratamentos especiais vem crescendo em indivíduos de diferentes faixas etárias. Aumentou o número de pacientes com doenças sistêmicas, sob medicação, idosos com dentes necessitando de uma odontologia adequada à sua situação, além do aumento das técnicas invasivas na odontologia, que implicam, também, um aumento do estresse no paciente e podem agravar uma doença sistêmica preexistente . $^{4}$

O grupo das DCNT compreende majoritariamente doenças cardiovasculares, diabetes, câncer e doenças respiratórias crônicas. Muitas doenças deste grupo têm fatores de risco comuns, e demandam por assistência continuada de serviços e ônus progressivo, na razão direta do envelhecimento dos indivíduos e da população. Muitos pacientes que procuram o tratamento odontológico exibem condições sistêmicas que podem alterar o curso de seus problemas bucais, bem como a terapêutica oferecida. A idade avançada da média dos pacientes com DP também contribui para o aumento da probabilidade de uma doença sistêmica preexistente ${ }^{5,6}$. As doenças cardiovasculares permanecem sendo as principais causas de morte na população e, corroborado por diversos autores, a doença coronária é entre, as doenças cardiovasculares, a de maior expressão tanto no nível de prevalência quanto no de morbimortalidade. É representada por um amplo espectro clínico que varia desde a doença arterosclerótica assintomática, passando por quadros de angina estável até quadros mais graves de infarto agudo do miocárdio, sendo imprescindível a intervenção cautelosa do cirurgião-dentista ${ }^{7}$.

Sendo assim, estudos descritivos têm contribuído na determinação de um padrão epidemiológico em uma população alvo, atribuindo certa previsibilidade nas alterações a serem encontradas, além de fornecer subsídios necessários para explicação de tais alterações ${ }^{8}$.
Diante do exposto e pela ainda reduzida oferta de revisões sobre o tema, o presente estudo visa analisar as alterações sistêmicas presentes nos prontuários dos pacientes atendidos na Clínica Odontológica do Centro Universitário Ruy Barbosa.

MATERIAL E MÉTODO

Para o desenvolvimento deste trabalho, foi realizado um levantamento dos prontuários dos pacientes atendidos, no período de 2016.2 a março de 2019, na Clínica de Odontologia do Centro Universitário Ruy Barbosa, totalizando 719 prontuários.

Os dados foram coletados a partir dos prontuários dos pacientes que se submeteram a atendimento no serviço de Odontologia da Faculdade e foram originalmente preenchidos pelos alunos de Graduação da instituição. Os prontuários preenchidos incorretamente ou com dados incompletos foram contabilizados como incompletos. Para a coleta e a tabulação dos dados, foi empregada uma tabela, sendo os mesmos dados analisados quantitativamente e transformados em porcentagem. Os fatores considerados para a avaliação incluíram: idade, gênero, condições de higiene bucal $e$ as principais enfermidades e condições sistêmicas que afetam a população mundial, tais como: hipertensão, doenças cardíacas, doenças endócrinas, doenças sanguíneas, doenças respiratórias, alergias medicamentosas e as alterações sistêmicas relatadas nos antecedentes familiares.

Os resultados foram analisados descritivamente, por porcentagem na presença de alterações sistêmicas dos pacientes encontrados no prontuário.

RESULTADOS

Foram analisados 719 prontuários, destes, 294 apresentaram respostas positivas a enfermidades ou condições sistêmicas. Alguns dos prontuários avaliados apresentaram mais de uma resposta positiva referente às mesmas. Pode-se observar que a hipertensão apresentou a maior prevalência com $81(27,55 \%)$ casos encontrados, seguido por anemia com 69 $(23,55 \%)$ casos, alergia medicamentosa com 41 $(17,9 \%)$ casos, diabetes e coagulopatia com 29 $(9,9 \%)$ casos cada um.

- Associação das alterações sistêmicas por faixa etária

Os pacientes foram divididos em sete faixas etárias, 13 - 19; 20 - 29; 30 - 39; 40 49; 50 - 59; 60 - 69 e 70 ou mais (tabela 1). Em ambos os gêneros, a maior prevalência de alteração sistêmica foi encontrada na faixa etária entre 40 a 49 anos, com 42 casos relatados no gênero feminino, correspondendo a 
$18,7 \%$ e 13 casos no gênero masculino, correspondendo a $18,85 \%$ deste grupo.

$\begin{array}{ll}\square \text { Hipertensão } & \square \text { Outros problemas cardiacos } \\ \square \text { Diabetes } & \square \text { Coagulopatia } \\ \square \text { Anemia } & \square \text { Alergia medicamentosa } \\ \square \text { Hipo/Hipertireoidismo } & \square \text { Outros problemas }\end{array}$

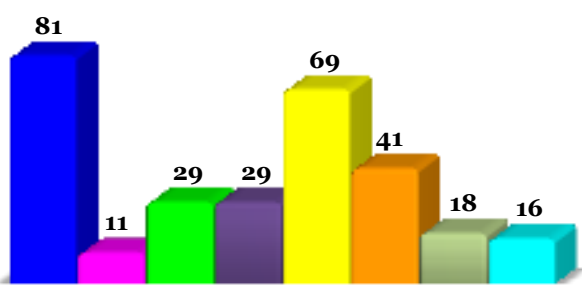

Figura 1; Condições sistêmicas mais prevalentes na população estudada (Fonte: Dados da pesquisa).

Tabela 1: Relação das alterações sistêmicas por faixa etária e gênero

\begin{tabular}{|l|c|c|}
\hline \multirow{2}{*}{ Faixa etária } & \multicolumn{2}{c|}{ Número de casos } \\
\hline 13 a 19 & Homens & Mulheres \\
\hline 20 a 29 & 7 & 24 \\
\hline 30 a 39 & 11 & 39 \\
\hline 40 a 49 & 8 & 38 \\
\hline 50 a 59 & 13 & 42 \\
\hline 60 a 69 & 10 & 28 \\
\hline 70 ou mais & 5 & 6 \\
\hline Sem informação & 7 & 13 \\
\hline Fonte: Dados da pesquisa & 8 & 35 \\
\hline
\end{tabular}

Tabela 2: Números de casos por alterações sistêmicas em diferentes faixas etárias

\begin{tabular}{|c|c|c|c|c|c|c|c|}
\hline $\begin{array}{l}\text { Alterações } \\
\text { sistêmicas }\end{array}$ & $\begin{array}{l}10 \text { a } \\
19\end{array}$ & $\begin{array}{l}20 \text { a } \\
29\end{array}$ & $\begin{array}{l}30 \text { a } \\
39\end{array}$ & $\begin{array}{l}40 a \\
49\end{array}$ & $\begin{array}{l}50 \text { a } \\
59\end{array}$ & $\begin{array}{l}60 \text { a } \\
69\end{array}$ & $\begin{array}{l}\text { 7o ou } \\
\text { mais }\end{array}$ \\
\hline Diabetes & o & 1 & 3 & 8 & 4 & 1 & 5 \\
\hline Hipertensão & 1 & 2 & 8 & 19 & 16 & 10 & 6 \\
\hline $\begin{array}{l}\text { Outras doenças } \\
\text { cardíacas }\end{array}$ & o & o & 1 & 1 & o & o & 2 \\
\hline Anemia & 22 & 12 & 8 & 14 & 8 & 0 & 0 \\
\hline $\begin{array}{l}\text { Alergia } \\
\text { medicamentosa }\end{array}$ & 7 & 11 & 9 & 9 & 2 & 1 & 3 \\
\hline Coagulopatia & 1 & 4 & 5 & 5 & 7 & 2 & 5 \\
\hline Hipo/Hipertireoidismo & 0 & 2 & 3 & 6 & 4 & o & o \\
\hline Outras doenç & 0 & 2 & 4 & 9 & 3 & 1 & 2 \\
\hline
\end{tabular}

Fonte: Dados da pesquisa

- Associação das alterações sistêmicas com o gênero

Dos $294(40,9 \%)$ casos positivos da afirmação da presença de alterações sistêmicas encontrados nos prontuários odontológicos, 225 $(76,5 \%)$ foram representados pelo gênero feminino e 69 (23,5\%) pacientes do gênero masculino e apresentaram alterações sistêmicas. Dos 225 pacientes do gênero feminino com alteração sistêmica analisados, a hipertensão apresentou a maior prevalência com 59 casos, correspondendo a 26,2\%; seguida por anemia com 56 casos, correspondendo a 24,9\%; alergia medicamentosa com 25 casos, correspondendo a $11,1 \%$ e a diabetes com 24 casos, correspondendo a $10,7 \%$ das enfermidades encontradas. Dos 69 pacientes do gênero masculino, a hipertensão apresentou uma maior prevalência com 22 casos encontrados, correspondendo a 31,9\%; seguida de alergia medicamentosa com 16 casos, correspondendo a 23,2\%; anemia com 13 casos, correspondendo a 18,8\%; coagulopatia com 6 casos, correspondendo a $8,7 \%$ das enfermidades encontradas.

Tabela 3: Prevalência das alterações sistêmicas encontradas no gênero feminino e masculino

\begin{tabular}{|l|c|c|}
\hline \multirow{2}{*}{ Alterações Sistêmicas } & \multicolumn{2}{c|}{ Número de Casos } \\
\hline Hipertensão & Homens & Mulheres \\
\hline Outros problemas cardíacos & 22 & 59 \\
\hline Diabetes & 3 & 8 \\
\hline Hipo/Hipertireoidismo & 5 & 24 \\
\hline Coagulopatia & 1 & 17 \\
\hline Anemia & 6 & 23 \\
\hline Alergia medicamentosa & 13 & 56 \\
\hline Outros problemas & 16 & 25 \\
\hline Fonte: Dados da pesquisa & 3 & 13 \\
\hline
\end{tabular}

\section{- Antecedentes familiares}

Em relação à avaliação dos antecedentes familiares, observou-se que 494 $(68,7 \%)$ dos pacientes relataram ter algum antecedente familiar com alterações sistêmicas. $\mathrm{Na}$ Tabela 4 observa-se a prevalência das alterações sistêmicas e enfermidades relatas pelos pacientes, entre seus familiares. A maior prevalência observada foi à hipertensão, com 373 casos listados correspondendo a $38,4 \%$ das alterações sistêmicas.

Tabela 4: Relação entre alterações sistêmicas e seus antecedentes familiares

\begin{tabular}{|l|c|c|}
\hline $\begin{array}{c}\text { Alteraç̃os sistêmicas } \\
\text { e enfermidades } \\
\text { encontradas }\end{array}$ & $\begin{array}{c}\text { Número de vezes } \\
\text { listadas }\end{array}$ & Percentual \% \\
\hline Hipertensão & 373 & $38,4 \%$ \\
\hline Outras doenças cardíacas & 100 & $10,3 \%$ \\
\hline Câncer & 108 & $11,1 \%$ \\
\hline Diabetes & 365 & $37,5 \%$ \\
\hline Hipotireoidismo & 3 & $0,3 \%$ \\
\hline Anemia & 7 & $0,7 \%$ \\
\hline Alzheimer & 2 & $0,2 \%$ \\
\hline AVE & 7 & $0,7 \%$ \\
\hline Aneurisma & 1 & $0,1 \%$ \\
\hline Trombose & 7 & $0,7 \%$ \\
\hline Fonte: Dados da pesquisa & & \\
\hline DISCUSSÃO & & \\
\hline
\end{tabular}

A importância da avaliação do paciente de uma maneira integrada passou a ser consenso em toda a área odontológica. Diante dessa aceitação, valorizou-se o estado sistêmico do paciente, o qual pode apresentar diversos distúrbios capazes de influenciar no tratamento odontológico proposto e comprometer o seu bem estar geral ${ }^{9}$.

Neste estudo foram avaliados 719 prontuários odontológicos, sendo encontrados 294 pacientes com respostas positivas para alterações sistêmicas. Esse resultado mostrou uma semelhança observada por outros autores quando comparada por percentual, onde verificou a presença de alterações sistêmicas em $49,06 \%$ de 693 prontuários no trabalho de 
Queiroz et al. ${ }^{10}$ e $44,13 \%$ de 4.330 prontuários avaliados Gaetti-Jardim et al. ${ }^{2}$. Divergindo do resultado do trabalho de Gadelha et al. ${ }^{4}$ que mostrou um número reduzido na prevalência de alterações sistêmicas, com apenas 6,62\% da população estudado com respostas positivas. Os autores afirmam que este dado pode ser explicado, principalmente, por omissão da condição sistêmica por parte dos pacientes, seja por ausência do conhecimento da condição clínica ou em virtude de medo de futuras intervenções médicas.

O gênero predominante com presença de alterações sistêmicas foi o gênero feminino, de certa forma as mulheres se preocupam mais com a saúde, priorizando uma boa qualidade de vida, dado este corroborado com Gadelha et al. ${ }^{4}$ e Queiroz et al. ${ }^{10}$. Contudo, no estudo de GaettiJardim et al. $^{2}$ o gênero mais predominante foi o masculino correspondendo a $64,57 \%$ dos pacientes. Estes autores justificam este achado pelo fato de seu trabalho ter sido realizado em âmbito hospitalar, o que é sabido, que o gênero masculino em que se verifica maior prevalência de fraturas faciais e denota maior prevalência nos politraumas.

Em relação a faixa etária por gênero com alterações sistêmicas, a mais prevalente foi entre 40 a 49 anos correspondendo ao gênero feminino, pois as alterações sistêmicas são mais propensas a se desenvolverem em indivíduos adultos, sendo assim, diferente do estudo de Gaetti-Jardim et al. ${ }^{2}$, onde a faixa etária mais acometida foi entre 20 a 29. No gênero masculino também houve divergência entre as pesquisas onde 0 presente estudo verifica que entre 40 a 49 houve uma maior prevalência, já na pesquisa de Gaetti-Jardim et al. $^{2}$ a faixa etária mais acometida foi a compreendida entre 31 a 40 anos de idade.

Dentre as alterações sistêmicas de maior prevalência, destacaram-se a hipertensão, sendo a mais prevalente entre as doenças cardiovasculares, e a diabetes sendo a mais prevalente entre as doenças endócrinas, mostrando semelhança no estudo de GaettiJardim et al. $^{2}$ onde a hipertensão e a diabetes mostraram-se as mais prevalentes dentre os grupos destas patologias. Esses dados podem ser justificados pelo fato destas doenças terem origem multifatorial, serem dependentes do estilo de vida do paciente, nutrição e até mesmo da hereditariedade e é sabido que com o avanço da tecnologia a vida sedentária da população aumentou, ampliando as chances do desenvolvimento de alterações sistêmicas.

Como também encontrado no estudo de Queiroz et al. ${ }^{10}$, pode-se observar casos de anemias, deixando claro que, esse tipo de alteração encontrada podem estar presente ou já ter tido esse tipo de manifestação ao decorrer da vida. A avaliação pré-operatória é de fundamental importância para o sucesso do tratamento, na medida em que quadros sistêmicos podem influenciar a indicação de um determinado procedimento e 0 tratamento odontológico proposto, comprometendo o bemestar do paciente. Atenção especial deve ser dada aos cuidados pré-operatórios que devem ser realizados nos pacientes comprometidos sistemicamente ${ }^{5}$

No estudo de Gaetti-Jardim et al. $^{2}$ as alergias apresentaram uma menor frequência, com apenas $11,08 \%$ dos casos, os autores relatam que esses dados podem refletir uma situação de negligência por parte dos profissionais, dificuldade de interpretação das respostas dadas pelos pacientes e, principalmente, pelos dados terem sido obtidos por várias pessoas diferentes dentro de um mesmo serviço da instituição, perdendo-se o processo de calibração dos analisadores. Já no presente estudo, a alergia apresentou uma prevalência maior que o estudo de GaettiJardim et al. $^{2}$, com $17,9 \%$ dos casos, esses dados podem sugerir a realização de forma minuciosa, sendo feito o questionamento a todos os pacientes avaliados.

Dentre os casos mencionados de alergia, pode-se observar relatos nos prontuários de alergia medicamentosa, que de acordo com Gomes et al. $^{11}$, as reações alérgicas a medicamentos podem envolver qualquer órgão ou sistema. Estas alterações podem modificar o plano de tratamento na hora da escolha da medicação a ser prescrita. Vale ressaltar a importância de uma boa anamnese e o conhecimento de sinais e sintomas de uma alergia medicamentosa para um futuro plano de tratamento. Dos medicamentos que mais causavam alergia, a dipirona foi a mais citada, seguida de anti-inflamatórios não esteroidais e penicilinas.

A utilização de medicamentos para o controle de manifestações sistêmicas, antecedentes familiares e o acompanhamento médico desses pacientes são fatores de suma importância para intervir clinicamente de forma segura ${ }^{4}$. No presente estudo pode-se observar que $36 \%$ dos pacientes com alterações sistêmicas fazem uso de medicamentos, confrontando com o estudo de Gadelha et al. ${ }^{4}$ onde $70,9 \%$ dos pacientes fazem uso de medicamentos, os dados no presente estudo podem ser explicados pelo fato de alguns pacientes apresentarem condições sistêmicas 
em estágios controladas apenas com a modificação do estilo de vida, ou pelo fato de alguns pacientes não fazerem 0 uso da medicação mesmo sendo recomendável, como pode ser observado em alguns relatos. A orientação para que estes pacientes façam o controle médico e medicamentoso das suas doenças é imprescindível para a manutenção do controle da sua saúde.

Os antecedentes familiares e a presença de alteração sistêmica são fatores predominantes para algumas alterações sistêmicas e 68,7\% dos pacientes deste trabalho relataram a presença de alteração sistêmica em um familiar de primeiro grau. Este resultado corrobora com o estudo feito por Gadelha et al. ${ }^{4}$ onde foram observadas $61,8 \%$ de respostas positivas para alterações sistêmicas em antecedentes familiares. Além disso, este estudo observou que a hipertensão e a diabetes apresentaram as maiores prevalências entre os familiares, o que justifica a incidência dos mesmos problemas de saúde presentes entre os pacientes, tendo em vista que a hipertensão arterial e o diabetes são duas doenças crônicas degenerativas e hereditárias. E reforça o papel do profissional de saúde nas orientações aos pacientes sobre os fatores de risco para desenvolvimento de certas doenças e, portanto, a necessidade com a prevenção.

Por fim, é de extrema importância que o cirurgião dentista realize uma minuciosa anamnese e um preenchimento completo do prontuário odontológico tendo em vista a saúde geral e bucal do paciente, para posteriormente o conhecimento sobre determinada alterações sistêmicas consequentemente suas manifestações bucais, a fim de evitar possíveis complicações e/ou acidentes durante ou após o tratamento odontológico.

CONCLUSÃO

As alterações mais prevalentes neste estudo foram a hipertensão, anemia, alergia e diabete e na maioria dos casos acometendo o gênero feminino. A faixa etária mais acometida foi entre 40 a 49 anos e o gênero mais prevalente é o feminino. Entre os antecedentes familiares, a hipertensão apresentou uma maior prevalência. Por isso é imprescindível um minucioso preenchimento do prontuário, destacando as alterações presentes para que seja determinado um plano de tratamento específico.

\section{REFERÊNCIAS}

1. Malta DC, Merhy EE. O percurso da linha do cuidado sob a perspectiva das doenças crônicas não transmissíveis. Interface. 2010; 14(34):593-605.

2. Gaetti-Jardim EC, Pereira FP, Fattah CMRS, Aranega AM. Prevalência e perfil epidemiológico das alterações sistêmicas em pacientes atendidos pelo serviço de cirurgia e traumatologia buco-maxilo-facial da Faculdade de Odontologia do Campus de Araçatuba UNESP. Rev Odontol UNESP. 2008;37(2): 191-96.

3. Chapper A, Goldadni MZ. A participação de odontólogos em equipes multidisciplinares. Revista Fac Odontol Porto Alegre 2004;45:3-5.

4. Gadelha LA, Silva RC, Lima VN, Momesso GAC, Queiroz SBF, Souza FA. Prevalência de doenças sistêmicas entre os pacientes atendidos na Clínica Odontológica da Faculdade Católica Rainha do Sertão no município de Quixadá-CE. Arch Health Invest. 2017;6(7):293-97

5. Brasil. Ministério da Saúde (MS). Secretaria de Vigilância em Saúde. Departamento de Análise de Situação de Saúde. Plano de ações estratégicas para o enfrentamento das doenças crônicas não transmissíveis (DCNT) no Brasil 2011-2022 Brasília, p. 30. 2011.

6. Guênes GMT, Guênes GT, Ribeiro AIAM, Dantas DCRE, Bento PM, Lins RDAU et al. Análise da condição periodontal e da necessidade de tratamento em pacientes cardiopatas. Scientia Medica. 2011;21(2): 49-54.

7. Kreuger MR, Diegoli NM, Pedrini RM, Porfério VP, Silva F. Consulta odontológica e doença sistêmica: análise do conhecimento dos cirurgiões dentistas em itajaí-sc. Rev Fac Odontol Lins. 2009;21(2):15-22.

8. Achutti A, Azambuka MIR. Doenças crônicas não-transmissíveis no Brasil: repercussões do modelo de atenção à saúde sobre a seguridade social. Ciênc saúde coletiva. 2004;9(4):833-40.

9. Menin C, Bortoloto FG, Farah GJ, Iwaki Filho L, Iwaki LCV, Leite PCC et al. Avaliação de pacientes hipertensos na clínica de cirurgia do terceiro ano do curso de odontologia do Cesumar. Iniciação Científica CESUMAR. 2006;8(2):147-56.

10. Queiroz TP, Marques DO, Santos PM, Saraiva $\mathrm{HC}$, Esteves JC, Vieira EH. Prevalência de alterações sistêmicas em pacientes atendidos na disciplina de Cirurgia e Traumatologia Bucomaxilofacial do curso de Odontologia da UNIARA. Rev Odontol UNESP. 2012;41(3): 154-59.

11. Gomes ER, Pichler WJ, Demoly P, Aberer, Frew AJ, Weck $A$, et al. The drug ambassador project: The diversity of diagnostic procedure for drug allergy around Europe. Allergy Clin Immunol Int - J World Allergy Org 2004; 17:9-18. 


\section{CONFLITO DE INTERESSES}

Os autores declaram não haver conflitos de interesse

\section{AUTOR PARA CORRESPONDENCIA}

Kaíque Carvalho de Souza

Curso de Odontologia, Centro Universitário Ruy

Barbosa Wyden - UNIRUY,

41730-101 Salvador - BA, Brasil

E-mail:kaique_nt@hotmail.com 